\title{
The Impact of CoRoT and Kepler on Eclipsing Binary Science
}

\author{
Carla Maceroni ${ }^{1}$, Davide Gandolfi ${ }^{2}$, Josefina Montalbán ${ }^{3}$, \\ and Conny Aerts ${ }^{4}$ \\ ${ }^{1}$ INAF-Osservatorio Astronomico di Roma, \\ via Frascati 33, I-00040, Monteporzio C. (RM), Italy \\ email: maceroni@oa-roma.inaf.it \\ ${ }^{2}$ ESA Estec, \\ Keplerlaan 1, 2201 AZ Noordwijk, Netherlands \\ email: dgandolf@rssd.esa.int \\ ${ }^{3}$ Institut d'Astrophysique et Géophysique Université de Liège, \\ Allée du 6 Aôut, B-4000 Liège, Belgium \\ $j$.montalban@ulg.ac.be \\ ${ }^{4}$ Institute of Astronomy, K.U.Leuven, \\ Celestijnenlaan 200D, B3001 Leuven, Belgium \\ email: conny@ster.kuleuven. be
}

\begin{abstract}
The CoRoT and Kepler space missions have opened a new era in eclipsing binary research. While specifically designed for exoplanet search, they offer as by-products the discovery and monitoring of variable stars, in great majority eclipsing binaries (EB). The missions are therefore providing thousands of EB light curves of unprecedented accuracy (typically a few hundred parts per million, ppm), with regular sampling (from $1^{\mathrm{s}}$ to $29^{\mathrm{m}}$ ), extending over time spans of months, and with a very high duty cycle (>90\%).

Thanks to this excellent photometry, research topics as asteroseismology of EB components are quickly developing, and physical phenomena such as doppler boosting, theoretically predicted but extremely difficult to observe from the ground, have been unambiguously detected. We present the main properties of the Corot and Kepler EB samples and briefly review the highlights of the missions in this field.
\end{abstract}

Keywords. binaries: eclipsing, stars: oscillations, surveys

\section{CoRoT and Kepler space missions}

CoRoT $\dagger$ (COnvection, ROtation and planetary Transits) is a French-led international "small" space mission launched in December 2006. The mission is devoted to the achievement of two parallel "core programs," asteroseismology and extra-solar planet search, which require the same type of observations, i.e. high accuracy photometry and long continuous monitoring. These programs are carried out in two contiguous "seismo" and "exo" fields, with a $27 \mathrm{~cm}$ telescope and four CCDs of $1.3^{\circ} \times 1.3^{\circ}$ on the sky. After launch each field was covered by two CCDs, the observations were performed in Long and Short runs lasting, respectively, $\sim 150$ and $\sim 30$ days, and for each run up to ten bright $(5.7>V>9.5)$ seismo-targets and up to 12000 exoplanet targets $(11.5>V>16.5)$ were observed. Unfortunately, the number of observed targets dropped to half after the loss in March 2009 of one of the two data processing units. That event induced as well a

$\dagger$ The CoRoT space mission was developed and is operated by the French space agency CNES, with participation of ESA's RSSD and Science Programmes, Austria, Belgium, Brazil, Germany, and Spain; complete information is available at http://corot.oamp.fr 
change in observing strategy (shorter "Long" runs of $\sim 80$ days have also been scheduled to increase the total number of targets).

CoRoT has provided high-accuracy $\left(10^{-3}-10^{-4} \mathrm{mag}\right)$ photometry of about 140,000 stars in a broad bandpass spanning $370-1000 \mathrm{~nm}$. Chromatic information was also obtained for exo-planet targets brighter than $V=15$.

Only a handful of EBs were observed in the seismo-field, as binaries are in general rejected in the target selection process. Sometimes, however, binarity is discovered by CoRoT itself (e.g., Maceroni et al. 2009). The standard sampling of the seismo field is $32^{\mathrm{s}}$, the fast one $1^{\mathrm{s}}$, yielding light curves with hundred thousands or millions of points and a point-to-point deviation of the order of $10^{-4}$ mag.

Most CoRoT EBs are exo-field targets, which are sampled at a standard rate of $8^{\mathrm{m}}$ or a fast one of $32^{\mathrm{s}}$. The light curves contain from 8000 to 300,000 points. An estimate of the white noise level as function of target R-magnitude (Aigrain et al. 2009) yields 0.5 mmag for $R=12$ and 2 mmag for $R=15$.

The classification of variable stars in the exo-fields is performed by the CoRoT Variability Classifier (CVC, Sarro et al. 2009, Debosscher et al. 2009), which provides a probabilistic classification in 29 different variability classes. Independent, and somehow different, lists of binaries have also been published for the first runs by the exoplanet search teams (Carpano et al. 2010, Cabrera et al. 2009, Carone et al. 2011) containing the EBs rejected by the planet search algorithms or subsequent follow-up observations.

So far nineteen different fields have been observed by CoRoT (and the data of the first ten are public $\dagger$ ), the results we present in this paper, however, refer mainly to $\sim 400 \mathrm{EBs}$ from the first CoRoT runs (IRa1, LRc1, LRa1) as for these fields EB samples from both CVC and exoplanet search are available.

CoRoT's trail is being widened by the Kepler space mission, thanks to its higher performance instrumentation and longer monitoring of targets. Kepler, NASA Discovery mission \#10, was specifically designed to discover Earth-size planets and is in operation since March 2009. The details of the mission can be found elsewhere (e.g., Borucki et al. 2010, Koch et al. 2010). In short, Kepler monitors 156,000 stars of interest (preferentially late type dwarfs) in a field of view extending over $105 \mathrm{deg}^{2}$ in the Cygnus Lyra region. The standard photometry sampling is $29.4^{\mathrm{m}}$ ("long cadence") but up to 512 stars can be observed in "short cadence" mode $\left(59^{\mathrm{s}}\right)$. The effective dynamic range is 7-17 Kepler magnitudes ( $\mathrm{K}_{p}$, in a broad bandpass from 425 to $\left.900 \mathrm{~nm}\right)$. The target continuous monitoring can last up to the mission lifetime (the programmed 3.5 years or longer in case of extension). The estimate of the instrument performance after launch (Koch et al. 2010) indicates that the design goal (a photometric precision of $20 \mathrm{ppm}$ for a $6.5 \mathrm{hr}$ exposure of a G2-type $\mathrm{V}=12$ target) is close to being achieved.

Kepler data are delivered in "Quarters" (Q0, Q1, .., Qn), typically three months long (a quarter ends when the spacecraft rolls to re-align its solar panels). Quarter Q10 is currently in progress, the first quarters (Q0-Q3) are publicly available $\ddagger$.

In addition to the exoplanet core program, scientific programs devoted to the asteroseismology of Kepler targets have been devised and outsourced to the European Kepler Asteroseismology Consortium (KASC), which maintains its own archive (KASOC). Subscription to this Consortium (open to collaboration) allows access to all the KASC data.

A comprehensive catalog of 1832 Kepler EBs, detected in the first two quarters Q0-Q1, has been published by Prša et al. (2011) and updated with the addition of Q2 data by Slawson et al. (2011). The current version contains 2165 eclipsing or ellipsoidal binaries.

$\dagger$ the Archive is mantained by the CoRoT Data Center at IAS, http://idoc-corot.ias.u-psud.fr/ $\ddagger$ available from the Multimission Archive at STScI (MAST) http://archive.stsci.edu/Kepler/ 

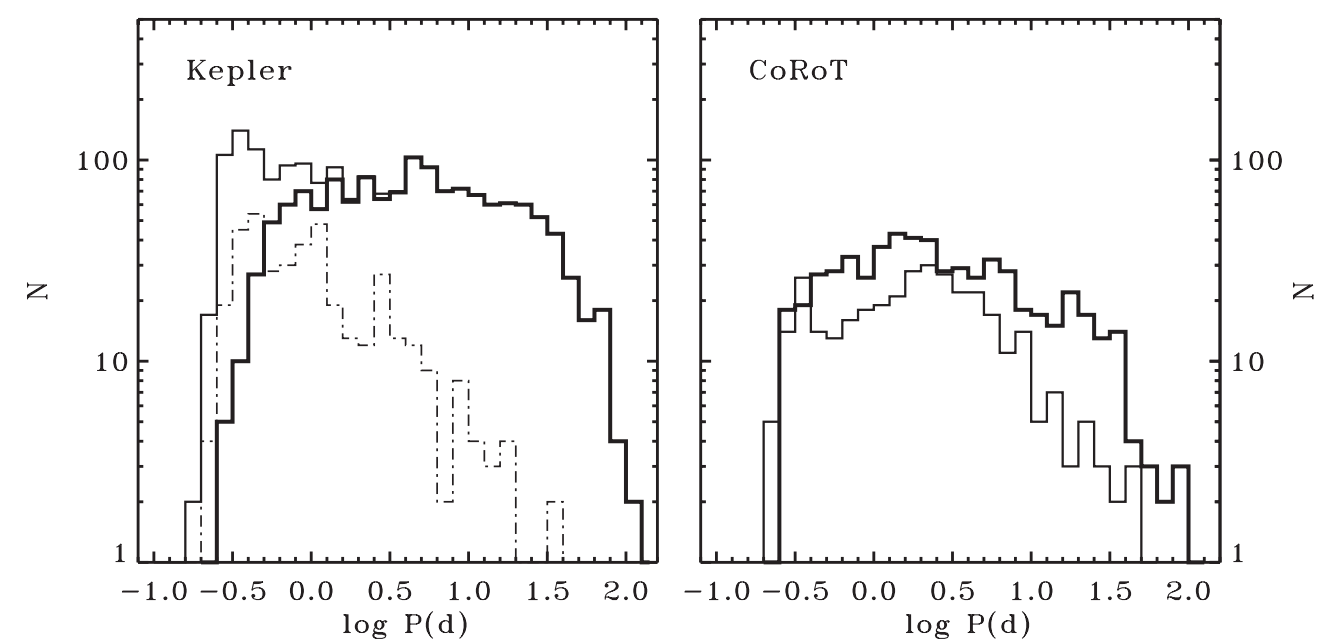

Figure 1. The period distribution of Kepler and CoRoT binaries. Left panel, thin line: all systems of Q0-Q2, thick line: detached and semidetached binaries only. For comparison the distribution of OGLE-I binaries with $\mathrm{I}<16.5$ is shown (dotted-dashed line), its quick decline is due to period dependent selection effects, affecting shorter periods for a ground based survey. Right panel, thin line: CVC sample (IRa1, LRa1, LRc1 fields), thick line: exoplanet sample for the same fields. The difference is related to the adopted filtering methods (see text).

\section{CoRoT and Kepler eclipsing binaries}

CoRoT and Kepler have provided light curves of unprecedented precision, sampling and extension. All these assets contribute to the increase of EBs frequency, which is about twice the value typical of ground-based surveys, as EBs with eclipses of smaller amplitude and shorter fractional eclipse duration are detected; see the comparison of CoRoT EB sample with that of OGLE-I in Maceroni (2010). The EB frequency of the first CoRoT fields is $1.2 \%$ of all targets. A similar, slightly larger, value of $1.4 \%$ is found by Slawson et al. (2011) for the first Kepler Quarters.

The higher efficiency of discovery of the space missions is evident as well in the orbital period distributions in Fig.1, showing different subsamples of EBs and a comparison with OGLE-I EBs of magnitude I > 16.5.

The difference between the CoRoT sample from CVC and that from exoplanet search is due to the different filter applied to extract binaries. The Fourier-based CVC algorithm fails more frequently for longer period narrow $\mathrm{V}$-shaped eclipses, especially if additional (quasi-) sinusoidal variability is present (the target is classified in another variability class). On the other hand the exo-planet algorithms, designed to detect U-shaped variations, misses short period (W UMa type) binaries. The CVC algorithm has been recently improved, to better handle binary extraction, so one can expect better agreement between the two sources for the following fields.

In both figures, and more markedly in the Kepler sample one, the log $\mathrm{P}$ distribution shows an almost flat behavior for periods larger than $1^{\mathrm{d}}$. Besides, the histograms suggest that the higher the precision, the more complete the sample for low amplitude detection, and the wider the "plateau" of the distribution. A flat $\log \mathrm{P}$ distribution, implying no preferred length scale for the formation of short-period binaries (Heacox 1998) is in agreement with the results of Mazeh et al. (2006) for a quite different sample (LMC B-type EBs). 


\section{CoRoT and Kepler highlights}

It is obviously impossible to concentrate in a few pages a comprehensive review of CoRoT and Kepler results of relevance to close and eclipsing binaries. However, already a few highlights will provide an idea of the outstanding achievements of both missions.

Eclipsing binaries with pulsating components. Most EBs light curves of Kepler and CoRoT present additional variability on the top of eclipses. This is often due to surface inhomogeneities (stellar activity) but another frequent cause is intrinsic stellar pulsation, which is monitored continuously and on long time spans. Pulsations undoubtedly make the analysis more complex, but add valuable information from an independent source. In a close binary, moreover, the influence of tides on surface stability can also be studied.

A good example is the case of CoRoT (seismo) target HD 174884 (Maceroni et al. 2009), an unusual eccentric eclipsing binary with twin B-type components but eclipse depths differing by factor of $\sim 100$. The analysis of the light curve of a few hundred ppm precision allowed to detect pulsations with amplitude of a few hundred ppm and frequencies exact multiples of the orbital one ( 8 and $13 f_{\text {orb }}$ ), which were interpreted as tidally excited pulsations.

Another interesting system is CoRoT 102918586, whose light curve is shown in Fig. 2 together with the Fourier spectrum after subtraction of the EB model, see Maceroni et al. (2010) for a description of the method. It is as well an example of the difficulties in the analysis when pulsations and eclipses are of comparable amplitude. The first analysis - presented in the above-mentioned paper and based on the CoRoT photometry alone - assumed a configuration with two very similar F0 dwarfs in a circular orbit and a period $P \simeq 8.78^{\mathrm{d}}$. The short fractional eclipse duration implied, however, very small fractional radii of the components (and no tidal deformation). On the other hand the light curve residuals, after subtraction of the binary model, contained harmonics of the orbital period, difficult to explain in terms of tidally excited pulsations, the stars being spherical and in circular orbit. The subsequent acquisition of high-resolution timeresolved spectroscopy has solved the issue: the true orbital period is half the value from photometry; the system is still formed by similar components (SB2) but the orbit is eccentric, and because of orientation in space only one eclipse is observed. The harmonics of the orbital period derived from the out of eclipse shape of the binary light curve, while
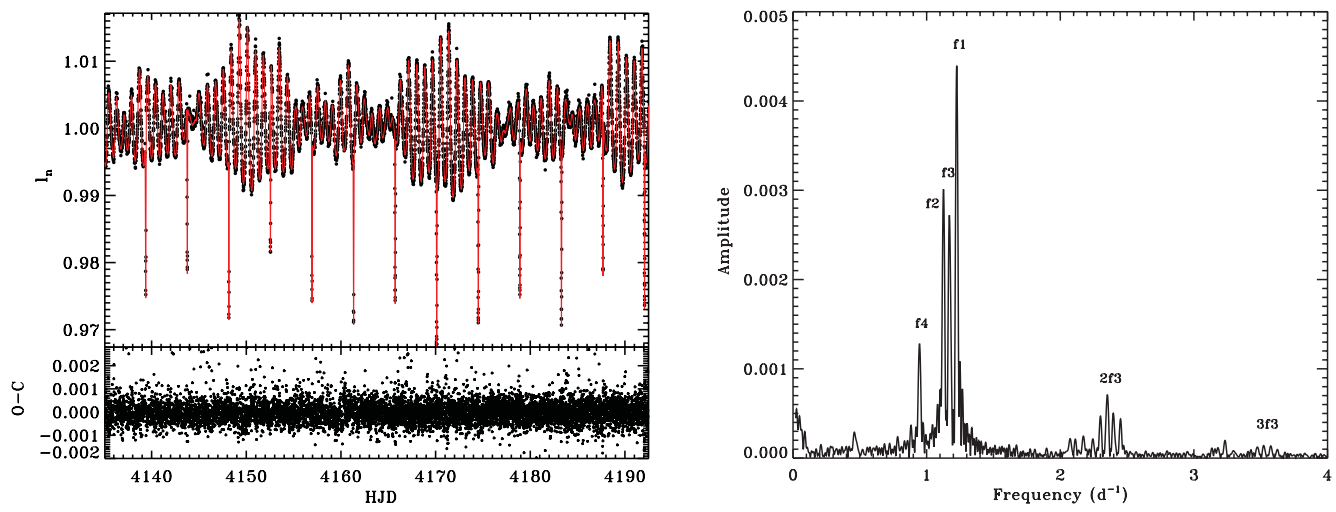

Figure 2. Left: The white-light lightcurve of CoRoT 102918586, time is in HJD-2450000, the continuous line is the fit (eclipsing binary model + pulsations), the lower box shows the corresponding residuals. Right: the power spectrum of the lightcurve after subtraction of the EB model. The main pattern (a multiplet of five frequencies around $f 3=1.1713 \mathrm{c} / \mathrm{d}$ ), repeats at $2 \times f 3$ and $3 \times f 3$. Frequency $f 4$ is $f 3-f_{\text {orb }}$. 
the analysis of the pulsations suggests the presence of a $\gamma$ Dor primary component and a rotational splitting of a $\ell=2$ gravity mode. A complete analysis of this interesting system will be presented elsewhere (Maceroni et al. 2011, in preparation).

In the case of solar-like pulsations (present as well in red giants) an estimate of "asteroseismic" mass and radius is possible based on the properties of the power spectrum (frequency of maximum power and large frequency separation) combined to effective temperature from spectroscopy and to scaling laws (Kjeldsen \& Bedding, 1995). This is especially valuable in the red giant case, where the estimate from classical methods is problematic. CoRoT has first detected solar-type oscillations in red giants (De Ridder et al. 2009), and among the first Kepler discoveries is that of a long period $\left(\sim 400^{\mathrm{d}}\right)$ eclipsing binary with a pulsating red giant component (Hekker et al. 2010), which is a promising milestone of such studies.

The Kepler satellite also implied the discovery of several compact binaries among its fast pulsators. Kawaler et al. (2010) found two compact binaries with gravity-mode pulsations superposed to an irradiation effect typical of sdB stars with a close M-dwarf companion. The orbital periods are less than half a day such that seismic sounding should become possible in the future, once the noise level of the data can be brought down to such a level that tidal and/or rotational splitting can be disentangled from period spacings of the modes. An even more interesting case is $2 \mathrm{M} 1938+4603$, an extremely rich pulsating $\mathrm{sdB}$ star in a $0.126^{\mathrm{d}}$ period binary with an eclipsing $\mathrm{dM}$ companion (Østensen et al. 2010). This star is a hybrid pulsator in that it reveals numerous pressure and gravity modes, offering the potential to probe both the outer layers and the inner core if the modes can be identified.

New discoveries: beaming binaries, tidal brightening. An exciting Kepler result is the clear detection of the relativistic beaming effect in the light curve of two targets: KPD 1946+4340 and KOI-74 (Bloemen et al. 2011, van Kerkwijk et al. 2010).

Beaming (also known as Doppler boosting) is a signature of the component radial velocity in the light curve. This takes the form of a modulation of measured flux according to the radial velocity difference, weighted by the component contribution to the total flux. It is, therefore, best observed in systems with components of very different spectral characteristics. When one star dominates the total flux the beaming effect measurement along the orbit provides information equivalent to that obtained from the radial velocity of a single lined spectroscopic binary.

The effect was predicted by Loeb \& Gaudi (2003) and Zucker et al. (2007) but its small amplitude (of the order of a few hundreds ppm for systems with periods of a few days) prevented detection in EBs before Kepler. For both detections the radial velocity amplitude from beaming is in excellent agreement with that derived from spectroscopy. KPD 1946+4340 and KOI-74 are the first members of the new class of "beaming binaries". The beaming effect has as well been detected in the light curve of CoRoT-3, a 22 Jupitermass object, orbiting an F3-star (Mazeh \& Faigler 2010).

Another remarkable, ever-observed, effect is the "tidal brightening" characterizing the light curve of KOI-54, a strongly eccentric $(e=0.83)$ non-eclipsing system with two similar A-type components and orbital period of $41.8^{\mathrm{d}}$ (Welsh et al. 2011). The light curve shows regular brightenings of a few mmag amplitude and tidally excited pulsations at high harmonics of the orbital frequency (90 and $91 \mathrm{f}_{\text {orb }}$ ). The orbital phenomena are interpreted as due to tidal distortion and irradiation at periastron. Several systems with brightenings and eclipses are found in the Kepler data and will provide new insights in tidal phenomena.

Tertiary eclipses. Finally, it is worth mentioning the Kepler discovery of a few triple (or multiple) systems showing tertiary eclipse events. The intersting case of HD 181068, a 
compact hierarchical triple system with a red giant component, has been fully analyzed by Derekas et al. (2011) and appears elsewhere in this volume. Another remarkable object, KOI-126 (Carter et al. 2011), shows eclipses from a closer pair formed by two M-dwarfs $\left(\mathrm{P}=1.7^{\mathrm{d}}\right)$ and from this close pair and a wider $1.35 \mathrm{M}_{\odot}$ third component $\left(\mathrm{P}=33.9^{\mathrm{d}}\right)$.

\section{Conclusions}

The exploitation of CoRoT and Kepler data will require many years, and, for sure, many exciting discoveries are still to come. The quality of the data is a formidable challenge for theoretical models and for the analysis tools, which have to be adapted to comply with the unprecedented accuracy of the data. Besides the excellent photometry has to be complemented by other observations (e.g., spectroscopy, interferometry, multicolor photometry) to take full advantage of its potentials, tasks requiring both time and manpower. The CoRoT community and KASC-WG9 (the working group on EBs) welcome collaboration to fully exploit these gold mines.

\section{References}

Aigrain, S., et al., 2009, A\&̈A, 506, 425

Bloemen, S., et al., 2011, MNRAS, 410, 1787

Borucki, W. J., et al., 2010, Science, 327, 977

Cabrera, J., et al., 2009, A\& $A$, 506, 501

Carpano, S., et al., 2009, A\&BA, 506, 491

Carone, L., et al., 2011, in preparation

Carter, J. A., et al., 2011, Science, 331, 562

Debosscher, J., et al., 2009, A\&GA, 506, 519

Derekas, A., et al., 2011, Science, 332, 216

De Ridder, J., et al., 2009, Nature, 459, 398

Heacox, W. D. 1998, AJ, 115, 325

Hekker, S., et al., 2010, ApJ, 713, L187

Kawaler, S. D., et al., 2010, MNRAS, 409, 1509

van Kerkwijk, M. H., Rappaport, S. A., Breton, R. P., Justham, S., Podsiadlowski, P., \& Han, Z. 2010, ApJ, 715, 51

Kjeldsen, H. \& Bedding, T. R. 1995, A\& A, 293, 87

Koch, D. G., et al., 2010, ApJ (Letters), 713, L79

Loeb, A. \& Gaudi, B. S. 2003, ApJ, 588, L117

Maceroni, C., et al., 2009, A\&BA, 508, 1375

Maceroni, C. 2010, ASP-CS, 435, 5

Maceroni, C., Cardini, D., Damiani, C., Gandolfi, D., Debosscher, J., Hatzes, A., Guenther, E. W., \& Aerts, C. 2010, arXiv 1004.1525

Mazeh, T., Tamuz, O., \& North, P. 2006, ApESSS, 304, 343

Mazeh, T. \& Faigler, S. 2010, A\&SA, 521, L59

Østensen, R. H., et al., 2010, MNRAS, 408, L51

Prša, A., et al., 2011, ApJ, 141, 83

Slawson, R. W., et al., 2011, arXiv 1103.1659

Sarro, L. M., Debosscher, J., López, M., \& Aerts, C. 2009, A\&A, 494, 739

Welsh, W. F., et al., 2011, arXiv 1102.1730

Zucker, S., Mazeh, T., \& Alexander, T. 2007, ApJ, 670, 1326 